\title{
Human Gait Gender Classification in Spatial and Temporal Reasoning
}

\author{
Kohei Arai ${ }^{1}$ \\ Graduate School of Science and Engineering \\ Saga University \\ Saga City, Japan
}

\author{
Rosa Andrie Asmara ${ }^{2}$ \\ Graduate School of Science and Engineering \\ Saga University \\ Saga City, Japan
}

\begin{abstract}
Biometrics technology already becomes one of many application needs for identification. Every organ in the human body might be used as an identification unit because they tend to be unique characteristics. Many researchers had their focus on human organ biometrics physical characteristics such as fingerprint, human face, palm print, eye iris, DNA, and even behavioral characteristics such as a way of talk, voice and gait walking. Human Gait as the recognition object is the famous biometrics system recently. One of the important advantage in this recognition compare to other is it does not require observed subject's attention and assistance. This paper proposed Gender classification using Human Gait video data. There are many human gait datasets created within the last 10 years. Some databases that widely used are University of South Florida (USF) Gait Dataset, Chinese Academy of Sciences (CASIA) Gait Dataset, and Southampton University (SOTON) Gait Dataset. This paper classifies human gender in Spatial Temporal reasoning using CASIA Gait Database. Using Support Vector Machine as a Classifier, the classification result is $97.63 \%$ accuracy.
\end{abstract}

Keywords- Gait Gender Classification; Gait Energy Motion; CASIA Gait Dataset.

\section{INTRODUCTION}

In recent years, there has been an increased attention on effectively identifying individuals for prevention of terrorist attacks. Many biometric technologies have emerged for identifying and verifying individuals by analyzing face, fingerprint, palm print, iris, gait or a combination of these traits [1][10][21]

Human Gait as the classification and recognition object is the famous biometrics system recently. Many researchers had focused this issue to consider for a new recognition system $[2][3][4][5][11][14][17][18][19][20]$ [21][24].

Human Gait classification and recognition giving some advantage compared to other recognition system. Gait classification system does not require observed subject's attention and assistance. It can also capture gait at a far distance without requiring physical information from subjects [3][4][5].

There is a significant difference between human gait and other biometrics classification. In human gait, we should use video data instead of using image data as other biometrics system used widely. In video data, we can utilize spatial data as well as temporal data compare to image data. Most of the gait classification and or recognition system created are using spatial data only[2][3][4][5][11][14][17][18][19][20][21][24].

Human Gait Gender Classification as a recognition system divided in three main subject; preprocessing, feature extraction and classification.

There are 2 feature extraction method to be used: model based and free model approach [2][20]. We used free modelbased for spatial data extraction and model-based for temporal data extraction. Model-based approaches obtain a set of static or dynamic skeleton parameters via modeling or tracking body components such as limbs, legs, arms and thighs. Gait signatures derived from these model parameters employed for identification and recognition of an individual. It is obvious that model-based approaches are view-invariant and scaleindependent. These advantages are significant for practical applications, because it is unlikely that reference sequences and test sequences taken from the same viewpoint. Model-free approaches focus on shapes of silhouettes or the entire movement of physical bodies. Model-free approaches are insensitive to the quality of silhouettes. Its advantage is a low computational costs comparing to model-based approaches. However, they are usually not robust to viewpoints and scale [3].

There are some Human Gait Datasets widely used by researchers today. Many human gait datasets created within the last 10 years. Some of widely used datasets are University of South Florida (USF) Gait Dataset, Chinese Academy of Sciences (CASIA) Gait Dataset, and Southampton University (SOTON) Gait Dataset. Our proposed method uses CASIA as a dataset resource. We used Class B of CASIA Dataset.

CASIA Class B is a large multi-view gait database, created in January 2005. There are 124 subjects, and the gait was captured from 11 views.

This paper will presents the classification of Human gait gender classification using our proposed method, Gait Energy Motion as a spatial feature and movement velocity as a temporal feature. The following section describes the proposed gender classification based on human gait followed by implementation and some experiments. Then conclusion with some discussions is also followed.

\section{PROPOSED METHOD}

The classification of human gait in this paper consists of three part, preprocessing, feature extraction, and classification. 
Figure 1 shows the complete overview of proposed human gait gender classification.
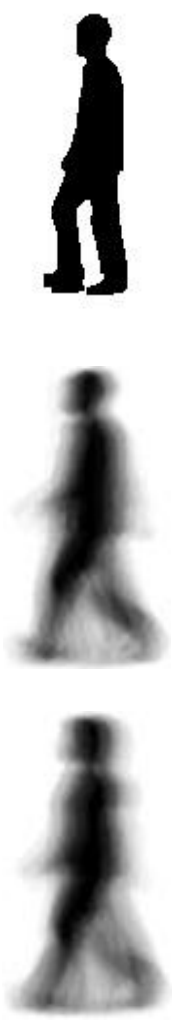

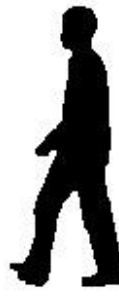

(a)

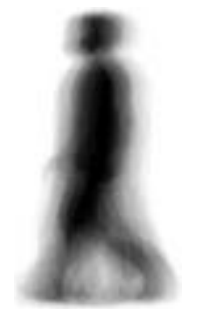

(b)

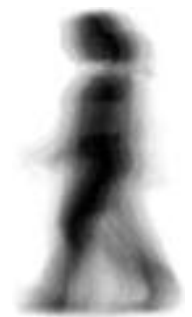

(c)
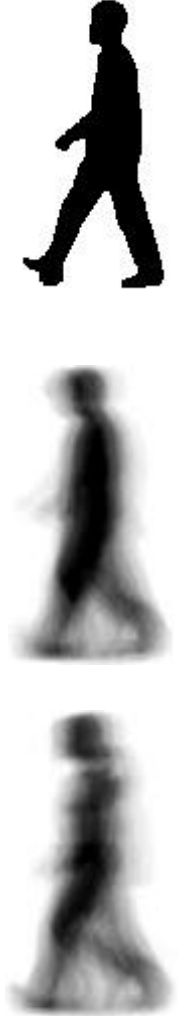

Figure 1(a). Silhouettes image (b). Male GEI image sample (c). Female GEI image sample

\section{A. Preprocessing}

In this proposed method, we use CASIA (Chinese Academy of Science) Gait Dataset of Class B using one perpendicular camera. The Class B Dataset consists of 124 different person. Each person had some conditions: bring bag, using coat, and normal condition. Each condition also taken some different angle camera position. The video resolution is 320 by 240 pixels, and using $25 \mathrm{fps}$ of frame rate. We use a normal condition and perpendicular camera position. First step preprocessing to get the feature is creating silhouettes, fortunately we did not have to create silhouettes because CASIA also provide silhouettes image in their dataset. Usually, silhouettes created using background subtraction.

\section{B. Feature Extraction}

We used free model-based for spatial data extraction and model-based for temporal data extraction.

\section{Free Model Based}

Free Model based using Gait Energy Image (GEI) is the best feature for gait recognition and classification until now[2][20]. GEI have some advantage compare to other feature. GEI represents all the silhouettes in one video file, into one new energy image. The dimensionality of the data is reduced using the energy. Using GEI will also remove the noise smoothly. It is said that GEI is a spatial temporal data, but in fact there are no temporal information in GEI. To overcome the temporal data, we use the model based which will be discuss in the next section.

Using GEI for gender classification also already published [20]. Instead of using raw GEI, Shiqi Yu et al. used some weighting point in some parts of the human body. The weighting factor achieved from some survey that their team taken in the research. The accuracy for gait gender classification using GEI and weighting point by Shiqi Yu et al. is $95.97 \%$.

Gender classification using gait motion as a feature also already done [26]. The gender classification accuracy is $92.9 \%$ using the gait motion.

To make comparative study with GEI, we will exploits motion feature as spatial data from human gait. Using the same method with GEI, but we just use the motion as a feature. We call this method Gait Energy Motion (GEM).

GEI is defined as [20] :

$$
F(i, j)=\frac{1}{T} \sum_{t=1}^{T} I(i, j, t)
$$

where $T$ is the number of frames in the sequence $I(i, j,$.$) ,$ $I(i, j, t)$ is a binary silhouette image at frame $t, i$, and $j$ are the image coordinates.

GEM is defined as :

$$
F(i, j)=\frac{1}{T} \sum_{t=2}^{T}|I(i, j, t)-I(i, j, t-1)|
$$

where $I(i, j, t)$ is a binary silhouette image at current frame $t$, and $I(i, j, t-1)$ is a binary silhouette image at previous frame $t$. Figure 2 shows the flowchart for generating GEM.

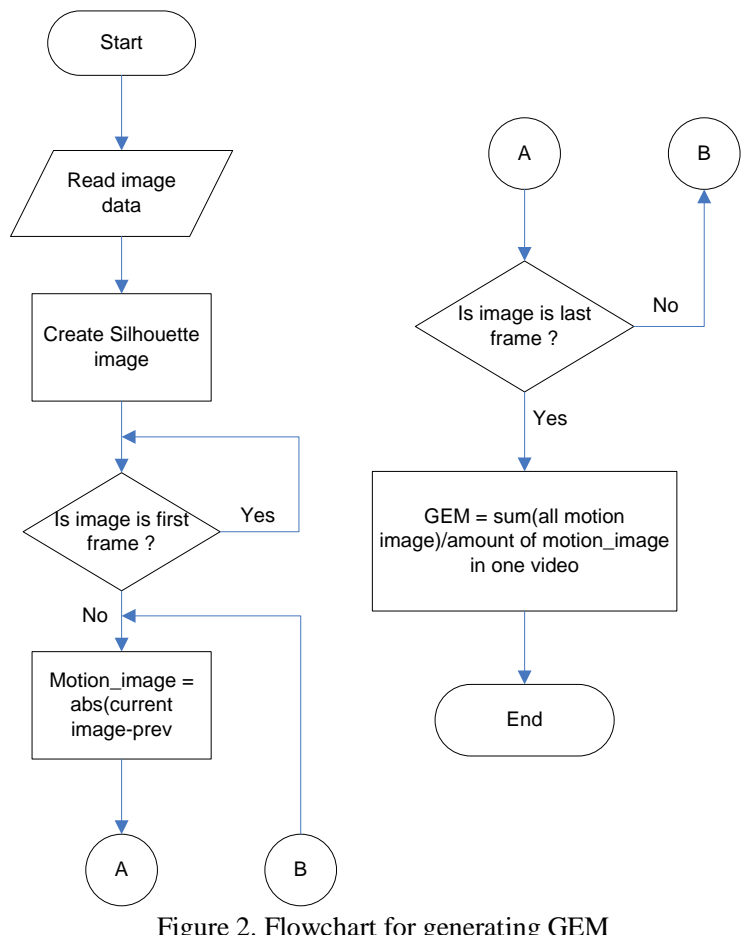

The model free preprocessing used in this paper by using the motion parameter per frame [22]. First, we have to get the silhouettes image. After we get the silhouettes, the motion of the human body can be achieved by using background 
subtraction. The motion we get also per frame and per video sequence. Figure 3 (a) is the results of the silhouettes image. Figure 3 (b) is the example of the human motion per frame. Figure 3 (c) is the example of Gait Energy Motion.

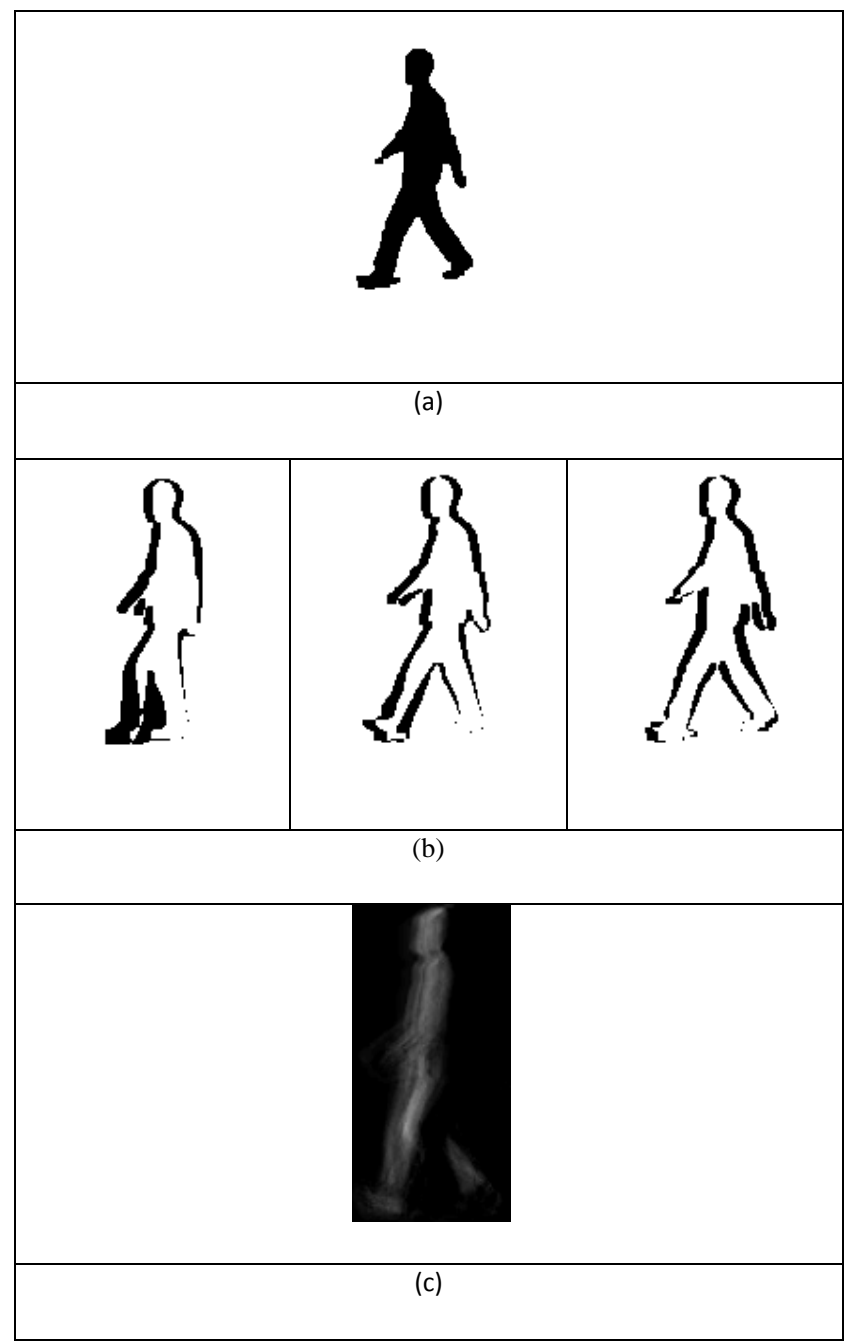

Figure 3. (a) Silhouettes image, (b) Motion image per frame, (c) Gait Energy Motion (GEM) result per video sequence.

Model based approaches obtain a series of static or dynamic body parameters via modelling or tracking body components such as limbs, legs, arms and thigh [2]. Model based approaches are view invariant and scale independent. Model based approaches are sensitive to the quality of gait sequences. Another disadvantage of the model based is its large computation and relatively high time costs due to parameters calculations.

Many gait model already made so far. We use M. S. Nixon et al. [4] model as a reference. This model provides more kinematics feature compare to other model. The challenge to this model is how to distinguish between left and right leg, because the data source only two-dimensional image using one perpendicular camera. This model can provide a lot of features, however, for the proposed method we start by only use one feature which is the velocity as a temporal data.
The methods namely Skeleton model. Morphological operation provides such a method to get skeleton image. The skeleton image having a lot of noise that can reduce the feature calculation result. For this research, we do not remove the noise because the we only used one temporal feature which is velocity.

We use three binary morphological operations to create such a skeleton model. Those three models are dilation, erosion, and thinning. For the dilation, we use three times of one's structuring element. We use six times of one's structuring element for the erosion.

Below is the algorithm for thinning operation [29]:

1. Divide the image into two distinct subfields in a checkerboard pattern.

2. In the first sub iteration, delete pixel $p$ from the first subfield if and only if the conditions $G_{1}, G_{2}$, and $G_{3}$ are all satisfied.

3. In the second sub iteration, delete pixel $p$ from the second subfield if and only if the conditions $G_{1}, G_{2}$, and $\mathrm{G}_{3}$ ' are all satisfied.

Condition $\mathrm{G}_{1}: \mathrm{X}_{\mathrm{H}}(\mathrm{p})=1$

(3)

where :

$$
\begin{gathered}
\mathrm{X}_{\mathrm{H}}(\mathrm{p})=\sum_{\mathrm{i}=1}^{4} \mathrm{~b}_{\mathrm{i}} \\
\mathrm{b}_{\mathrm{i}}=\left\{\begin{array}{l}
1 \text { if } x_{2 i-1}=0 \text { and }\left(x_{2 i}=1 \text { or } x_{2 i+1}=1\right) \\
0 \text { otherwise }
\end{array}\right. \\
\text { Condition } \mathrm{G}_{2}: 2 \leq \min \left\{n_{1}(p), n_{2}(p)\right\} \leq 3
\end{gathered}
$$

where :

$\mathrm{n}_{1}(\mathrm{p})=\sum_{\mathrm{k}=1}^{4} \mathrm{x}_{2 \mathrm{k}-1} \vee \mathrm{x}_{2 \mathrm{k}}$
$\mathrm{n}_{2}(\mathrm{p})=\sum_{\mathrm{k}=1}^{4} \mathrm{x}_{2 \mathrm{k}} \vee \mathrm{x}_{2 \mathrm{k}+1}$

$$
\begin{aligned}
& \text { Condition G3: }\left(x_{2} \vee x_{3} \vee \overline{x_{8}}\right) \wedge x_{1}=0 \\
& \text { Condition G3': }\left(x_{6} \vee x_{7} \vee \overline{x_{4}}\right) \wedge x_{5}=0
\end{aligned}
$$

Figure 4 shows the result of silhouettes after some morphological operation proposed.

We can calculate the velocity easily using head point in every frame as a reference. The unit measure for velocity in this method is pixel/frame.

\section{IMPLEMENTATION AND EXPERIMENTS}

We will implement the proposed methods to the CASIA (Chinese Academy of Sciences) Gait Database. CASIA Gait dataset has four class datasets: Dataset A, Dataset B (multiview dataset), Dataset C (infrared dataset), and Dataset D (foot pressure measurement dataset). We will use the $\mathrm{B}$ class dataset 
in 90 degrees point of view. Figure 8 shows the CASIA Gait image database example of male and female gender [9].
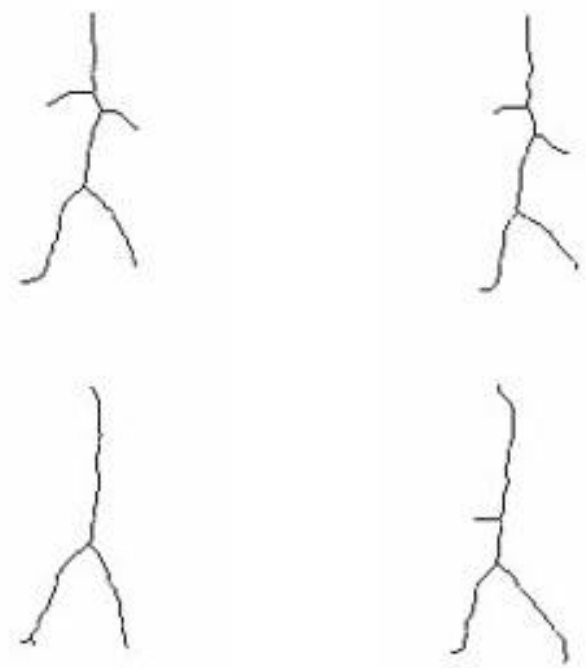

Figure 4. Skeleton after some morphological operation
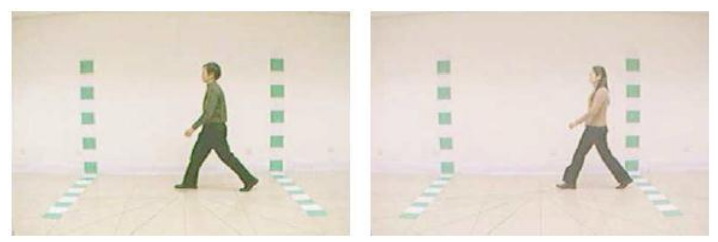

Figure 5. CASIA Gait image example of male and female

There are 124 unique human gaits in B class dataset. From the total of 124 dataset, only 31 data of female gait existed. To make the data balance, we will also use 31 male gait dataset. Every human has 6 video of perpendicular view, so we pretend to use every single video as one data. The total of the data is 372 , consists of 186 video of male gait and 186 video of female gait.

We use Support Vector Machine: SVM as a classification method and using 10 cross fold validation as a training method. We also try the well-known Two Dimensional: 2D Discrete Wavelet Transformation: DWT with Haar base function for analyzing and comparing the results [26]. The result is in Table 1 . Very interesting to analyze is the classification time for every method. Every pixel is pretend to be the feature want to classify. The classification cost will be higher if the image size is bigger. GEM is smaller image compare to GEI, because GEM only took the motion part, not all part of the image. Using Approximation detail in DWT will reduce the classification cost because the image size is rescaling four times smaller in level 1. Smaller image size will also reduce the CCR.

Since we use Gait Energy Motion (GEM) which is taking the motion parameter in the image, the difference between classes should be the motion. Also another basic question is which part of the human body giving the significance difference between classes.
TABLE I. CCR TABLE FOR SOME METHODS ANALYZED

\begin{tabular}{|l|c|c|}
\hline \multicolumn{1}{|c|}{ Method } & $\begin{array}{c}\text { Classification } \\
\text { Time }\end{array}$ & CCR \\
\hline $\begin{array}{l}\text { Gait Energy Image (GEI) with } \\
\text { some weighting factor [20] }\end{array}$ & $1078.5 \mathrm{~ms}$ & $95.97 \%$ \\
\hline Gait Energy Motion (GEM) & $722.2 \mathrm{~ms}$ & $97.47 \%$ \\
\hline GEM with Velocity & $\mathbf{7 4 4 . 5} \mathbf{~ m s}$ & $\mathbf{9 7 . 6 3 \%}$ \\
\hline $\begin{array}{l}\text { 2D DWT GEM Lv 1. Using } \\
\text { Approximation Coefficient }\end{array}$ & $67.1 \mathrm{~ms}$ & $97.32 \%$ \\
\hline $\begin{array}{l}\text { 2D DWT GEM Lv 1. Using } \\
\text { Velocity }\end{array}$ & & \\
\hline
\end{tabular}

In [20], Shiqi Yu et al. used analysis of variance (ANOVA) F-statistics to analyze the gait difference between classes. We will use the same method to analyze GEM difference. The ANOVA F-statistic is a measure to evaluate different features discriminative capability. The greater the Fstatistic values will give better discriminative capability. The F-statistic is calculated as follows [20]:

$F=\frac{\frac{1}{c-1} \sum_{i=1}^{c} n_{i}\left(\bar{x}_{i}-\bar{x}\right)^{2}}{\frac{1}{n-c} \sum_{i=1}^{c} \sum_{j=1}^{n_{i}}\left(x_{i j}-\bar{x}_{i}\right)^{2}}$

where $x_{i j}$ is the $j^{\text {th }}$ sample of class $i, c$ is the number of classes, $n_{i}$ is the sample number of class $i, n=\sum_{i=1}^{c} n_{i}, \bar{x}_{i}$ is the mean of samples in class $i$, and $\bar{x}$ is the mean of $\bar{x}_{i}$. If we implement in our research, the formula above will become:

$\mathrm{F}=$

$180\left(\left(\text { mean }_{\text {gem_male }}-\text { mean }_{\text {gem_all }}\right)^{2}+\left(\text { mean }_{\text {gem_female }}-\text { mean }_{\text {gem_all }}\right)\right)^{2}$ $\frac{1}{370}\left(\sum\left(\text { gem }_{\text {male }(\mathrm{i}, \mathrm{j})}-\text { mean }_{\text {gem_male }}\right)^{2}+\sum\left(\text { gem }_{\text {female }(\mathrm{i}, \mathrm{j})}-\text { mean }_{\text {gem_female }}\right)^{2}\right)$

The calculated F-statistic values are shown in Figure. Whiter color means better discriminative capability. The highest discriminative value is seen in the left foot motion. Right foot motion also seen some discriminative value, but because of the longer distance than the left foot, the value is not too high. Other areas that have higher discriminative value than others are the hand motion.

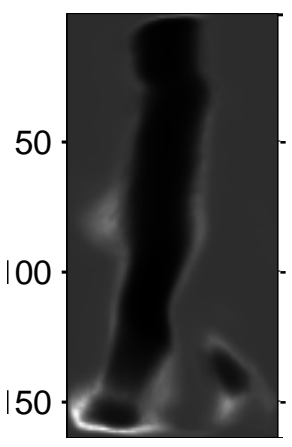

Figure 6. F-Statistics image 
TABLE II. CCR FOR SOME PUBLISHED METHODS

\begin{tabular}{|l|l|l|}
\hline Method & Dataset & CCR \\
\hline Lee and Grimson & 25 males \& 25 females & $85.0 \%$ \\
\hline Huang and Wang [27] & 25 males \& 25 females & $85.0 \%$ \\
\hline Li et al. [19] & 31 males \& 31 females & $93.28 \%$ \\
\hline 44 human observers & 31 males \& 31 females & $95.47 \%$ \\
\hline Shiqi Yu et al. [20] & 31 males \& 31 females & $95.97 \%$ \\
\hline Proposed method & 31 males \& 31 females & $97.63 \%$ \\
\hline
\end{tabular}

\section{CONCLUSION}

The entire system is using model free motion based and model motion based as spatial and temporal information. GEM as a spatial feature and velocity as a temporal feature extraction, and classify the data using SVM. Implemented in the CASIA Gait Database, we conclude as following:

(1) The classification accuracy is $97.47 \%$ if only spatial information processed.

(2) The classification accuracy is $97.63 \%$ if spatial and temporal information processed,

This research shows that GEM is giving more CCR result compare to GEI with some weighting factors. This research also shows that by using free model motion based and velocity gait is enough to use as a feature for human gait gender classification. We even do not need any weighting point factors in the feature to increase the classification result.

The preprocessing used in this proposed method is a model free based. There are some advantages by using this method. First, the development of the program is not difficult. Because it is not too difficult, another advantage of this method is low cost computation system.

We use velocity from a model based feature to get temporal information. This feature proved enough to classify gender. However, for human recognition using gait, we should use other kinematics parameter from a model that we created. There is some temporal information such as stride and cadence parameter per frame, angle of every knee per frame, and hip angle per frame. Some other classification might provide a better result to the recognition of human gait gender such as a k-nearest neighbor [14].

\section{ACKNOWLEDGMENT}

Portions of the research in this paper use the CASIA Gait Database collected by Institute of Automation, Chinese Academy of Sciences. In his connection, authors would like to thank to Chinese Academy of Sciences for their providing of the Gait database.

\section{REFERENCES}

[1] X. Qinghan, "Technology review - Biometrics Technology, Application, Challenge, and Computational Intelligence Solutions", IEEE Computational Intelligence Magazine, vol. 2, pp. 5-25, 2007.
[2] Jin Wang, Mary She, Saeid Nahavandi, Abbas Kouzani, "A Review of Vision-based Gait Recognition Methods for Human Identification", IEEE Computer Society, 2010 International Con-ference on Digital Image Computing: Techniques and Applications, pp. 320 - 327, 2010

[3] N. V. Boulgouris, D. Hatzinakos, and K. N. Plataniotis, "Gait recognition: a challenging signal processing technology for biometric identification", IEEE Signal Processing Magazine, vol. 22, pp. 78-90, 2005.

[4] M. S. Nixon and J. N. Carter, "Automatic Recognition by Gait", Proceedings of the IEEE, vol. 94, pp. 2013-2024, 2006.

[5] Y. Jang-Hee, H. Doosung, M. Ki-Young, and M. S. Nixon, "Automated Human Recognition by Gait using Neural Network", in First Workshops on Image Processing Theory, Tools and Applications, 2008, pp. 1-6.

[6] Wilfrid Taylor Dempster, George R. L. Gaughran, "Properties of Body Segments Based on Size and Weight", American Journal of Anatomy, Volume 120, Issue 1, pages 33-54, January 1967

[7] Gilbert Strang and Truong Nguen, "Wavelets and Filter Banks". Wellesley-Cambridge Press, MA, 1997, pp. 174-220, 365-382

[8] I. Daubechies, Ten lectures on wavelets, Philadelphis, PA : SIAM, 1992.

[9] CASIA Gait Database, http://www.cbsr.ia.ac.cn/ english/index.asp

[10] Edward WONG Kie Yih, G. Sainarayanan, Ali Chekima, "Palmprint Based Biometric System: A Comparative Study on Discrete Cosine Transform Energy, Wavelet Transform Energy and SobelCode Methods", Biomedical Soft Computing and Human Sciences, Vol.14, No.1, pp.1119, 2009

[11] Dong Xu, Shuicheng Yan, Dacheng Tao, Stephen Lin, and Hong-Jiang Zhang, Marginal Fisher Analysis and Its Variants for Human Gait Recognition and Content- Based Image Retrieval, IEEE Transactions On Image Processing, Vol. 16, No. 11, November 2007

[12] Hui-Yu Huang, Shih-Hsu Chang, A lossless data hiding based on discrete Haar wavelet transform, 10th IEEE International Conference on Computer and Information Technology, 2010

[13] Kiyoharu Okagaki, Kenichi Takahashi, Hiroaki Ueda, Robustness Evaluation of Digital Watermarking Based on Discrete Wavelet Transform, Sixth International Conference on Intelligent Information Hiding and Multimedia Signal Processing, 2010

[14] Bogdan Pogorelc, Matjaž Gams, Medically Driven Data Mining Application: Recognition of Health Problems from Gait Patterns of Elderly, IEEE International Conference on Data Mining Workshops, 2010

[15] B.L. Gunjal, R.R.Manthalkar, Discrete Wavelet Transform based Strongly Robust Watermarking Scheme for Information Hiding in Digital Images, Third International Conference on Emerging Trends in Engineering and Technology, 2010

[16] Turghunjan Abdukirim, Koichi Niijima, Shigeru Takano, Design Of Biorthogonal Wavelet Filters Using Dyadic Lifting Scheme, Bulletin of Informatics and Cybernetics Research Association of Statistical Sciences, Vol.37, 2005

[17] Seungsuk Ha, Youngjoon Han, Hernsoo Hahn, Adaptive Gait Pattern Generation of Biped Robot based on Human's Gait Pattern Analysis, World Academy of Science, Engineering and Technology 342007

[18] Maodi Hu, Yunhong Wang, Zhaoxiang Zhang and Yiding Wang, Combining Spatial and Temporal Information for Gait Based Gender Classification, International Conference on Pattern Recognition 2010

[19] Xuelong Li, Stephen J. Maybank, Shuicheng Yan, Dacheng Tao, and Dong $\mathrm{Xu}$, Gait Components and Their Application to Gender Recognition, IEEE Transactions On Systems, Man, And CyberneticsPart C: Applications And Reviews, Vol. 38, No. 2, March 2008

[20] Shiqi Yu, , Tieniu Tan, Kaiqi Huang, Kui Jia, Xinyu Wu, A Study on Gait-Based Gender Classification, IEEE Transactions On Image Processing, Vol. 18, No. 8, August 2009

[21] M.Hanmandlu, R.Bhupesh Gupta, Farrukh Sayeed, A.Q.Ansari, An Experimental Study of different Features for Face Recognition, International Conference on Communication Systems and Network Technologies, 2011

[22] S. Handri, S. Nomura, K. Nakamura, Determination of Age and Gender Based on Features of Human Motion Using AdaBoost Algorithms, 2011

[23] Massimo Piccardi, Background Subtraction Techniques: Review, http://www-staff.it.uts.edu.au/ $\sim$ massimo/BackgroundSubtractionReview-Piccardi.pdf

[24] Rosa Asmara, Achmad Basuki, Kohei Arai, A Review of Chinese Academy of Sciences (CASIA) Gait Database As a Human Gait Recognition Dataset, published in the Industrial Electronics Seminar 2011, Surabaya Indonesia 
[25] Bakshi, B., "Multiscale PCA with application to MSPC monitoring," AIChE J., 44, pp. 1596-1610., 1998

[26] Kohei Arai, Rosa Andrie, "Human Gait Gender Classification using 2D Discrete Wavelet Transforms Energy", IJCSNS International Journal of Computer Science and Network Security, Vol. 11 No. 12 pp. 62-68, December 2011

[27] G. Huang, Y. Wang, "Gender classification based on fusion of multiview gait sequences," in Proc. $8^{\text {th }}$ Asian Conf. Computer Vision, 2007, pp. 462-471.

[28] L. Lee, WEL Grimson, "Gait analysis for recognition and classification," in Proc. $5^{\text {th }}$ IEEE Int. Conf. Automatic Face and Gesture Recognition, Washington, DC, May 2002, pp. 155-162.

AUTHORS PROFILE

Kohei Arai received BS, MS and PhD degrees in 1972, 1974 and 1982, respectively. He was with The Institute for Industrial Science and Technology of the University of Tokyo from April 1974 to December 1978 and also was with National Space Development Agency of Japan from January, 1979 to
March, 1990. During from 1985 to 1987, he was with Canada Centre for Remote Sensing as a Post-Doctoral Fellow of National Science and Engineering Research Council of Canada. He moved to Saga University as a Professor in Department of Information Science on April 1990. He was a counselor for the Aeronautics and Space related to the Technology Committee of the Ministry of Science and Technology during from 1998 to 2000. He was a councilor of Saga University for 2002 and 2003. He also was an executive councilor for the Remote Sensing Society of Japan for 2003 to 2005 . He is an Adjunct Professor of University of Arizona, USA since 1998. He also is Vice Chairman of the Commission A of ICSU/COSPAR since 2008. He wrote 29 books and published 352 journal papers.

Rosa A. Asmara received the B.E. degree in electronics engineering from Brawijaya University, and the M.S. degree in Multimedia engineering, from Institute of Technology Sepuluh Nopember, Surabaya, Indonesia, in 2004 and 2009 , respectively. He is currently a PhD Student at Information Science in Saga University, Japan. His research interests include signal processing, image processing, parallel processing, pattern recognition, and computer vision 